ISSN 1991- 8690

website : http:// jsci.utq.edu.iq

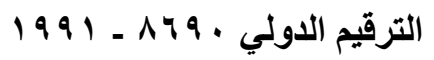

Email: utjsci@utq.edu.iq

\title{
Dynamical behavior of quantum-dot laser
}

\author{
M.O. Oleiwi \\ C.A. Emshary
}

Physics Department - Education College for Pure Sciences - Basrah University

\begin{abstract}
$\underline{\text { Abstract }}$
In this work we have investigated the dynamical properties of InAs/InGaAs laser emitting $1.3 \mu \mathrm{m}$ pumped by injection current density of fast rise and falling times with a duration of $5 n s$. In (capture) and out (escape) scattering rates of electrons and holes are studied against temperature, injection current density, Auger coefficient .Scattering times are studied against carrier reservoir loss coefficient, injection current density and temperature. Temporal behavior of photons density against injection current density is given too.
\end{abstract}

\section{التصرف الحركي لليزر النقطة الكمية}

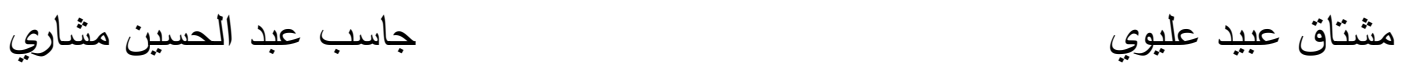

$$
\text { قسم الفيزياء - كلية التربية للعلوم الصرفة - جامعة البصرة }
$$

المستخلص

في هذا العمل درسنا الخصائص الحركية لليزر النقطة الكمية InAs/InGaAs الباعث للضوء بطول موجي 1.3 والذي يضخ بكثافة تيار حقن ذا زمني ارتقاء وهبوط سريعين وبطول (5ns) .تم استقصاء المعدلات الزمنية لاستطارتي الاقتتاص والهروب لكل من الالكترونات والفجوات بتغير

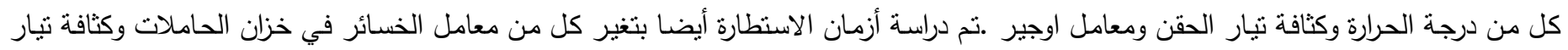
الحقن ودرجة الحرارة درسنا أيضا التصرف الزمني لكثافة الفوتونات بتغير كثافة تيار الحقن.

\section{$\underline{\text { Introduction }}$}

In this work we study overall dynamics of In $\mathrm{As} / \mathrm{InGaAs}$ quantum dot laser such as photon density and number of electrons and holes in QD (against injection current density), the in and out scattering rates between the QD and WL(against temperature, injection current density and Auger coefficient ) and number of electrons and holes in WL (against temperature and Auger coefficient ). The three-dimensional confinement of electrons and holes in a semiconductor quantum dot
(QD) profoundly changes the density of state compared to a bulk semiconductor or thin -film quantum well (QW). In ensembles of QDs, the ideal delta- function density of states of a single dot is modified into a nearly Gaussian contour that is determined by the degree of inhomogeneity in the QD sizes and shapes. QD lasers have attracted much attention in recent years due to their superior properties, such as ultra -low and temperature -stable threshold current density, high speed operation, and low frequency chirping [1]. In this paper we investigate the performance of QD 
semiconductor laser by considering a two level system of $\hbar \omega=0.96 \mathrm{eV}$ as a common for selforganized QDs InAs/InGaAs material system. The carrier-carrier scattering rates for electron and hole capture into the QD levels $S_{e}{ }^{i n}$ and $S_{h}^{i n}$ and those for carrier escape from the QD levels $S_{e}{ }^{\text {out }}$ and $S_{h}{ }^{\text {out }}$ , the scattering times for electrons $\tau_{e}$ and holes $\tau_{h}$ carrier densities in wetting layer $w_{e}$ and $w_{h}$, the Auger coefficient $B_{A}$, the shift of the device temperature inside the laser medium $(T)$, a carriers densities in the QD and photon density are studies versus Auger coefficient, current density and time .

\section{QD Laser Model:}

The numerical investigations of the laser turn-on dynamics of the QD laser presented here are based on the model given by Kathy Ludge et.al [2-5]. In the QD laser system the electrons are first injected into the wetting WL before they are captured by the QDs. The laser dynamics is determined by the rate equations for the photon density $n_{p h}$ of the ground state, GS, transition, and carrier densities in the QD, $n_{e}$ and $n_{h}$ and the carrier densities in the WL, $w_{e}$ and $w_{h}$ ( $e$ and $h$ stand for electrons and holes, respectively) this model reads :

$$
\begin{aligned}
& \dot{n}_{p k}=-2 k n_{p h}+\Gamma R_{i n d}\left(n_{\varepsilon}, n_{h}, n_{p h}\right)+\beta R_{s p}\left(n_{\varepsilon}, n_{h}\right) \\
& \dot{n_{s}}=-\frac{1}{\tau_{\varepsilon}} n_{\varepsilon}+S_{\varepsilon}^{i n} N^{\varrho D}-\Gamma R_{\text {ind }}\left(n_{\varepsilon}, n_{h}, n_{p h}\right)-R_{s p}\left(n_{\varepsilon}, n_{h}\right) \\
& \dot{n}_{k}=-\frac{1}{\tau_{h}} n_{h}+S_{h}^{i n} N^{\varrho D}-\Gamma R_{\text {ind }}\left(n_{\varepsilon}, n_{h}, n_{p h}\right)-R_{s p}\left(n_{\varepsilon}, n_{h}\right) \\
& \dot{w}_{\varepsilon}=\eta \frac{j(t)}{e_{\theta}}+\frac{n_{\varepsilon}}{\tau_{\varepsilon}} \frac{N^{s u m}}{N^{Q D}}-S_{\varepsilon}^{\text {in }} N^{s u m}-\tilde{R}_{s p}\left(w_{\varepsilon}, w_{h}\right) \\
& \dot{w}_{h}=\eta \frac{j(t)}{e_{0}}+\frac{n_{h}}{\tau_{h}} \frac{N^{s v n}}{N^{Q D}}-S_{h}^{i n} N^{s v n}-\tilde{R}_{s p}\left(w_{e}, w_{h}\right)
\end{aligned}
$$

$R_{\text {ind }}\left(n_{e}, n_{h}, n_{p h}\right)=W A\left(n_{e}+n_{h}-N^{Q D}\right) n_{p h} \quad$ is the linear gain, $N^{Q D}$ denotes twice the QD density of the lasing subgroup (the factor of 2 accounts for spin degeneracy), $W$ is the Einstein coefficient, and $A$ is the WL normalization area $(A=4 \mu m \times 1 \mathrm{~mm})$. The density $N^{\text {sum }}$ is twice the total QD density. The spontaneous emission in the QDs is approximated by $R_{s p}\left(n_{e}, n_{h}\right)=\left(W / N^{Q D}\right) n_{e} n_{h}[1]$.

$\tilde{R}_{s p}\left(w_{e}, w_{h}\right)=B^{S} w_{e} w_{h}$ expresses the WL spontaneous recombination rate where $B^{S}$ is the bandband recombination coefficient in the WL. $\beta$ is the spontaneous emission coefficient and $\Gamma=\Gamma_{g} N^{Q D} / N^{\text {sum }}$ is the optical confinement factor. $\Gamma$ is the product of the geometric confinement factor $\Gamma_{g}$ ( i.e the ratio of the volume of all QDs and the mode volume) and the ratio $N^{Q D} / N^{\text {sum }}$. The total cavity loss is expressed by $2 \kappa$. The variable $j(t)$ is the injection current density, $e_{o}$ is the electronic charge, and $\eta=1-w_{e} / N^{W L}$ is the injection efficiency that accounts for the fact that we cannot inject any more carriers if the WL is already filled $\left(w_{e}=N^{W L}\right)$. A sketch of the epitaxial structure as well as the energy diagram of the band structure is shown in Fig. (1) [4].

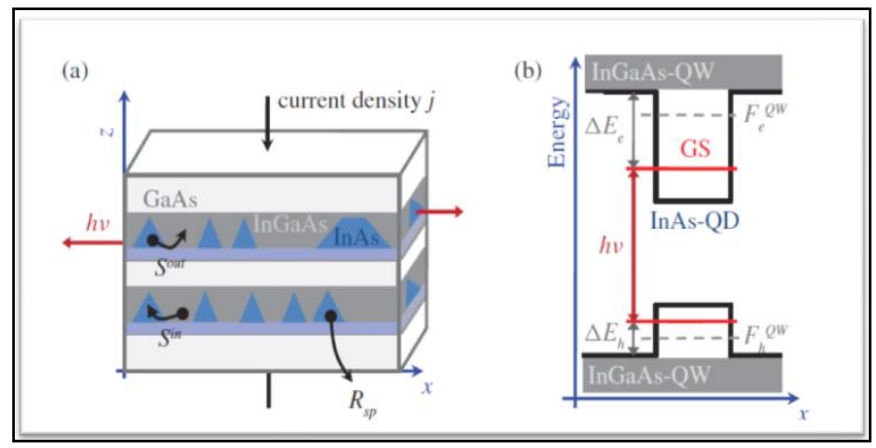

Fig. 1. (a) Schematic illustration of the QD laser. (b) Energy diagram of the band structure across a QD. $h v$ labels the ground state (GS) lasing energy. $\Delta E_{e}$ and $\Delta E_{h}$ mark the distance( in energy) of the GS from the QW band edge for electrons and holes, respectively. $F_{e}^{Q W}$ and $F_{h}^{Q W}$ are the quasi-Fermi levels for electrons and holes in the QW, respectively [4].

The spectral properties of the laser output are not addressed in the model, as the photon density is an average over all longitudinal modes. Changes in the QD size distribution are taken into account only by changes in the active QD density, which 
basically changes the gain. The values of parameters used in our simulations are listed in Table 1. A crucial contribution to the dynamics of QD lasers is given by the nonradiative carriercarrier scattering rates (nonlinear scattering rates) $S_{e}^{i n}$ and $S_{h}^{i n}$ for electron and hole capture into the QD levels, $S_{e}{ }^{\text {out }}$ and $S_{h}{ }^{\text {out }}$ for carrier escape from the QD levels, and scattering times $\tau_{e}=\left(S_{e}^{\text {in }}+S_{e}^{\text {out }}\right)^{-1}$ and $\tau_{h}=\left(S_{h}^{\text {in }}+S_{h}{ }^{\text {out }}\right)^{-1}$. These rates are determined microscopically within the Boltzmann equation and orthogonalized planewave approach [6]. All electron-electron, holehole, and mixed electron-hole Auger processes are included in the rates [7]. The WL carrier density is very high, the capture dynamics within the QDWL structure is dominated by Coulomb scattering (nonlocal Auger recombination).The calculated scattering rates depend in a strongly nonlinear way upon the WL carrier densities [6], [7].

Table 1 :'Numerical parameters used in the simulation

\begin{tabular}{lllll}
\hline symbol & value & & symbol & value \\
\cline { 1 - 2 }$W$ & $0.7 \mathrm{~ns}^{-1}$ & & $A$ & $4 \times 10^{-5} \mathrm{~cm}^{2}$ \\
$T$ & $300 \mathrm{~K}$ & & $N^{Q D}$ & $0.6 \times 10^{10} \mathrm{~cm}^{-2}$ \\
$2 \kappa$ & $0.1 \mathrm{ps}^{-1}$ & & $N^{\text {sum }}$ & $20 \times 10^{10} \mathrm{~cm}^{-2}$ \\
$\Gamma_{g}$ & 0.075 & & $N^{W L}$ & $2 \times 10^{13} \mathrm{~cm}^{-2}$ \\
$\Gamma$ & $2.25 \times 10^{-3}$ & & $B^{S}$ & $850 \mathrm{~ns}^{-1} \mathrm{~nm}^{2}$ \\
$\beta$ & $5 \times 10^{-6}$ & & $m_{e}\left(m_{h}\right)$ & $0.043(0.45) m_{0}$ \\
$\Delta E_{e}$ & $190 \mathrm{meV}$ & & $\Delta E_{h}$ & $69 \mathrm{meV}$ \\
\hline \hline
\end{tabular}

The scattering times for escape and capture for the carriers defined as $\tau_{b}{ }^{c a p}=1 / S_{b}{ }^{i n}$ and $\tau_{b}{ }^{\text {esc }}=1 / S_{b}{ }^{\text {out }}(b=e, h) \quad$ can be expressed as [2] : $\tau_{h}{ }^{c a p}=\frac{1.5 p s . n m^{-2}}{538 n m^{2} w_{h}{ }^{2}+w_{h}}+6 p s$

$\tau_{h}{ }^{e s c}=\frac{0.24 p s . n m^{-2}}{100 n m^{2} w_{h}{ }^{2}+w_{h}}+86 p s$

$\tau_{h}=\frac{0.29 p s . n m^{-2}}{w_{h}}-3.6 p s$

$\tau_{e}^{c a p} \approx \tau_{e}=\frac{5.2 p s . n m^{-2}}{w_{e}+1570 \mathrm{~nm}^{2} w_{e}{ }^{2}}+27 p s$
It can be seen that for the hole scattering time, the dependence on $w_{h}$ is close to what would be expected with the assumption of a linear scattering rate. In contrast to this, researchers find a more complicated functional relation for the electron scattering rate. It has to be noted that for even higher WL carrier densities, both electron and hole scattering times will finally increase due to Pauli blocking of the scattered Auger electron [1].

\section{Turn-on Characteristics:}

To depict the measured laser output for different pump currents $j$ (given in units of the laser threshold $j_{t h}$, which is determined from the simulated steadystate input-output characteristic $n_{p h}(j)$. The injection current pulse with a duration of $5 n s$ is switched on at $(\mathrm{t}$ $=0$ ). The results of the simulation is shown in Fig.(2) . For the simulation a current pulse $j(t)=j_{0} \exp \left[-\left(\frac{t-t_{0}}{2.5 n s}\right)^{n}\right]$ with $t_{0}=2.49 \mathrm{~ns}$ and $n=90$ is used, yielding a flat plateau $j=j_{0}$ with rise and fall times of $100 \mathrm{ps}$ [Fig. (3)].

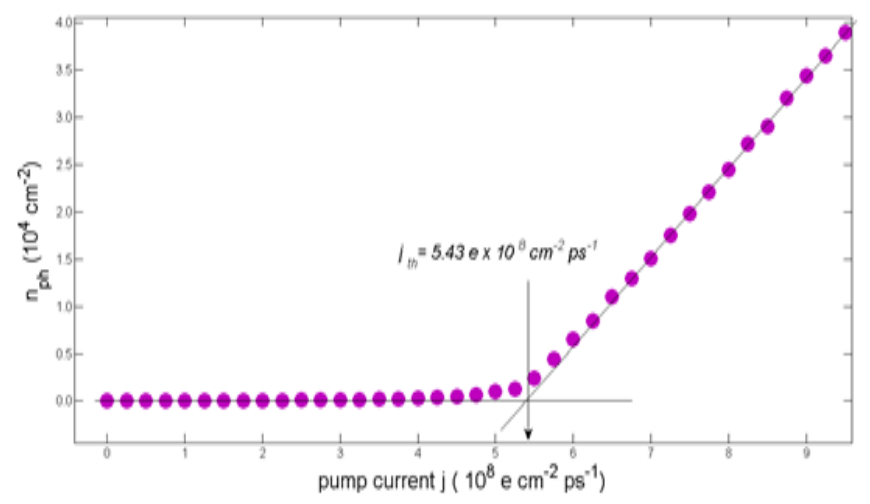

Fig. (2) Steady-state input-output characteristic: simulated photon density $n_{p h}$ vs. injection current density $j$. The threshold current density $j_{t h}=5.43 e_{0} \times 10^{8} \mathrm{~cm}^{-2} \mathrm{ps}^{-1}$ is determined from the extrapolated laser onset if spontaneous emission is neglected. Parameters as in Table 1. 


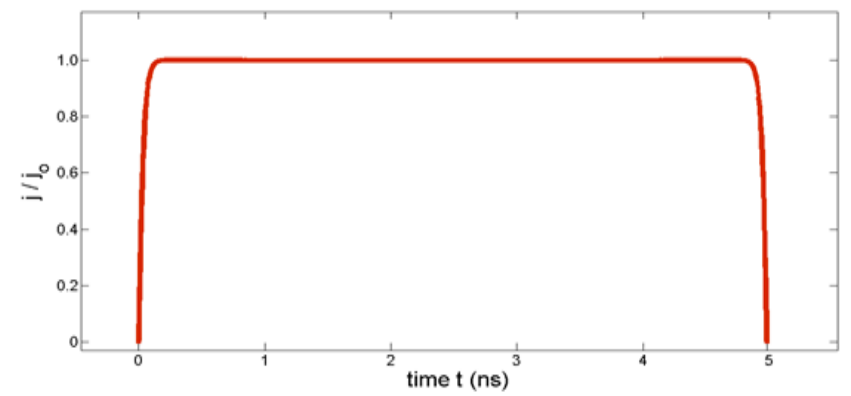

Fig.(3). Injection current pulse used in the simulations of turn-on characteristics

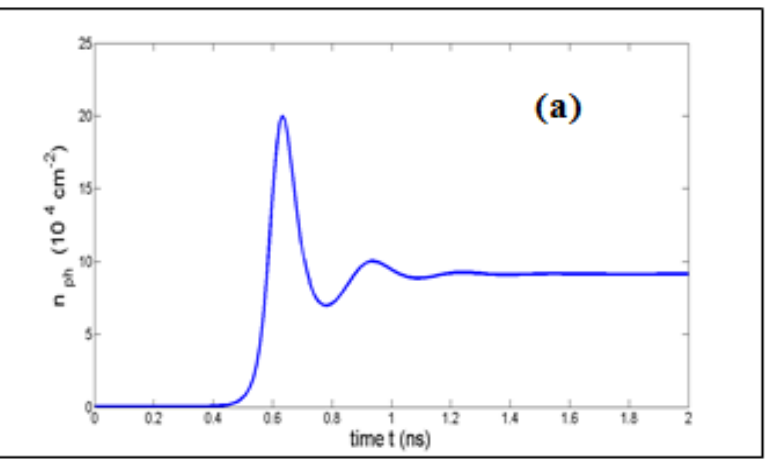

The simulation results of photon density in the QD laser are obtained by solving the set of equations (1-5) using the fourth order Runge - Kutta numerical integration method with Matlab .The results are shown in fig.(4) at constant injection current density are studied for different injection current densities(the result are shown in Fig.(5)).

Fig.(4) Simulation of the temporal variation of (a) photon density $n_{p h}$ and (b)electrons(upper) and holes (lower) densities in the $Q D\left(n_{e}, n_{h}\right)$, respectively, for injection current density $\left(j=2.7 j_{t h}\right)$.
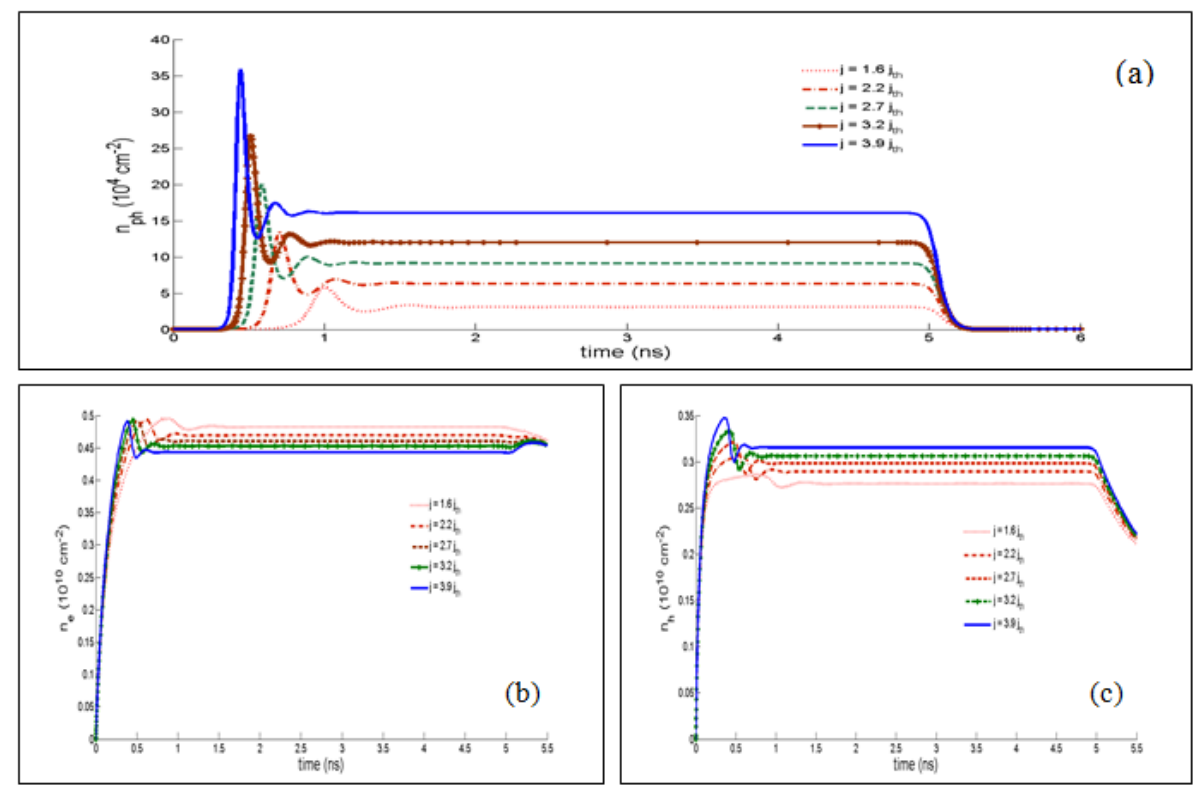

Fig.(5) Simulation of the temporal variation of (a) photon density $n_{p h}$, (b) electron density $n_{e}$ and (c) hole density $n_{h}$ in the QD for different injection current densities $j=(1.6,2.2,2 \cdot 7,3.2,3.9)$ 


\section{Temperature-Dependent Losses in the Reservoir:}

The temperature , $\mathrm{T}$, dependence of the in- and outscattering rates, the carrier losses inside the reservoir can modeled as a function of $T$. The effect of these $T$ dependent losses will be most prominent for the large signal response of the laser while its effect on the turnon dynamics and modulation response is small. The rate $R_{\text {loss }}=B\left(w_{e}\right) w_{e} w_{h}$ that accounts for these losses is a sum of the spontaneous bimolecular band-band recombination and Auger-related losses inside the quantum well (or wetting layer) $\mathrm{QW}$ given by $B_{A} w_{e} w_{h}$. The Auger coefficient $B_{A}$ has been shown [8] to depend significantly on the temperature $T$, and is therefore implemented such that it leads to a doubling of the rate for a temperature change of $60 \mathrm{~K}$ as found in [1]. Thus, $B_{A}=305 \mathrm{~nm}^{4} \mathrm{ps}^{-1}\left(\frac{T}{300 K}\right)^{4}$ is used as given in [4]. Keep in mind that in this work a laser with only GS levels in the QDs is modeled, which results in a different $B_{A}$ for the remaining Auger processes within the QW. An alternative approach to model temperature characteristics is described by M. Rossetti et. al [9] by assuming nonradiative losses in the reservoir, which are modeled by capture processes from the reservoir to a mid gap defect level. The Auger

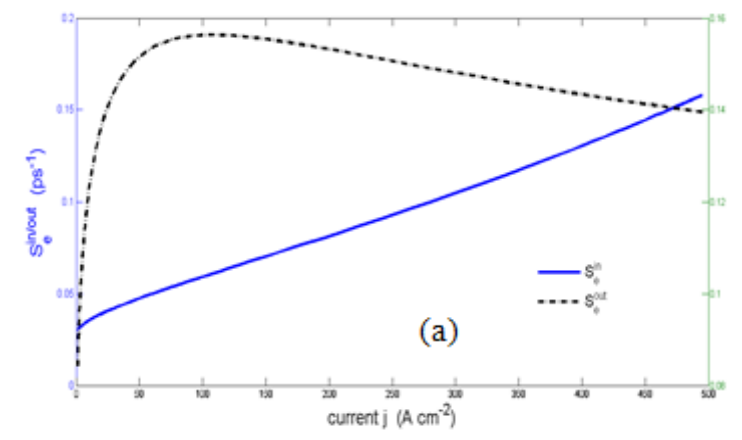

scattering rates depend on the carrier temperature inside the QW. The following analytic expressions in order to allow for an implementation into the rate equations are used [4]:

$$
\begin{aligned}
& S_{e}^{\text {in }}\left(T, w_{e}, w_{h}\right)=(1+0.22(T-300) / 100 K) \cdot S_{e}^{\text {in }}\left(300, w_{e}, w_{h}\right) \\
& S_{h}^{\text {in }}\left(T, w_{e}, w_{h}\right)=(1+0.22(T-300) / 100 K) \cdot S_{h}^{\text {in }}\left(300, w_{e}, w_{h}\right)
\end{aligned}
$$

The out-scattering rates are related to the in-scattering rates by detailed balance as derived in [2] and [5]:

$$
\begin{gathered}
S_{\varepsilon}{ }^{\text {out }}\left(T, w_{\varepsilon}, w_{h}\right)=S_{\varepsilon}{ }^{i n}\left(T, w_{\varepsilon}, w_{h}\right) \cdot \frac{e^{K T}}{e^{\frac{w_{\varepsilon}}{\rho_{\varepsilon} K T}}-1} \\
S_{h}{ }^{\text {out }}\left(T, w_{\varepsilon}, w_{h}\right)=S_{h}{ }^{i n}\left(T, w_{\varepsilon}, w_{h}\right) \cdot \frac{e^{-\frac{\Delta E_{k}}{K T}}}{e^{\frac{w_{k}}{\rho_{k} K T}}-1}
\end{gathered}
$$

Here, $\Delta E_{e}$ and $\Delta E_{h}$ are the energy separations between the QD electron and hole GS and the lowest respective QW state (see fig.(1)) $\rho_{e}=m_{e} / \pi \hbar^{2} \quad$ and $\rho_{h}=m_{h} / \pi \hbar^{2}$ are the respective 2-D densities of state in the QW, $m_{e}$ and $m_{h}$ are the electron and hole masses respectively. As the injection current density increased so does the in- scattering rates to then QD while the out-scattering of electrons and holes shows optimum values then decreases, see Fig.(6).

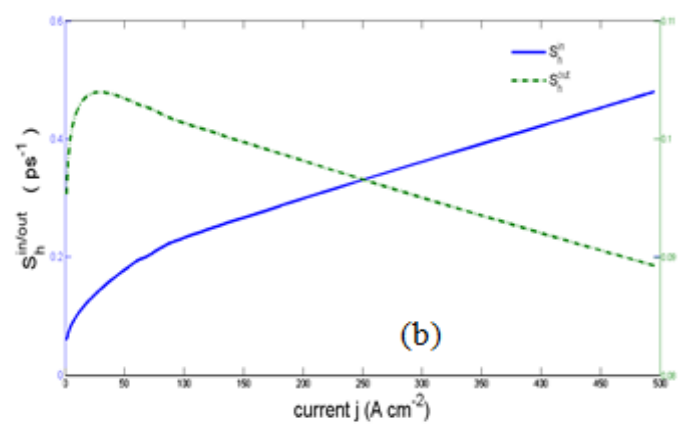

Fig.(6) Simulation results of variation of (a) scattering rates of electrons $\left(S_{e}{ }^{\text {in }}, S_{e}^{\text {out }}\right)$ against injection current density $(j)$ and (b)scattering rates of holes $\left(S_{h}{ }^{\text {in }}, S_{h}{ }^{\text {out }}\right)$ against injection current density $(j)$.

As the temperature change between 0 and $325 \mathrm{~K}$ the relations between $S_{e, h}{ }^{\text {in }}$ and $S_{e, h}{ }^{\text {out }}$ and Auger coefficient are shown in Fig.(7) together with the direct relation between the temporal $\mathrm{T}$ and scattering rates . 

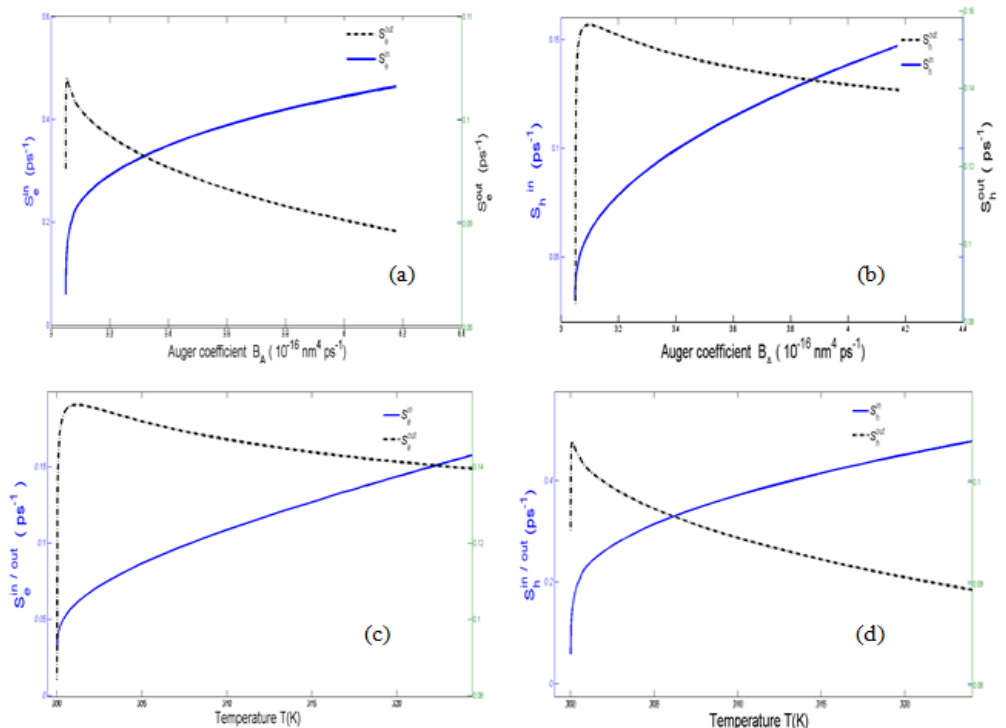

Fig.(7) (a, b) : Simulation result of variation of the results of scattering rates of electrons and holes $\left(S_{b}{ }^{i n}, S_{b}{ }^{\text {out }}\right)$, against Auger coefficient $B_{A}$ and (c,d) : simulation result of variation of scattering rates of $\left(S_{b}{ }^{\text {in }}, S_{b}{ }^{\text {out }}\right)$ against temperature $T(K), b=e, h$.

As a consequence of the result shown in Fig.(7), the and holes respectively, are shown in Fig.(8). behavior of scattering times, ${ }^{\tau_{e}}$ and $\tau_{h}$ of electrons
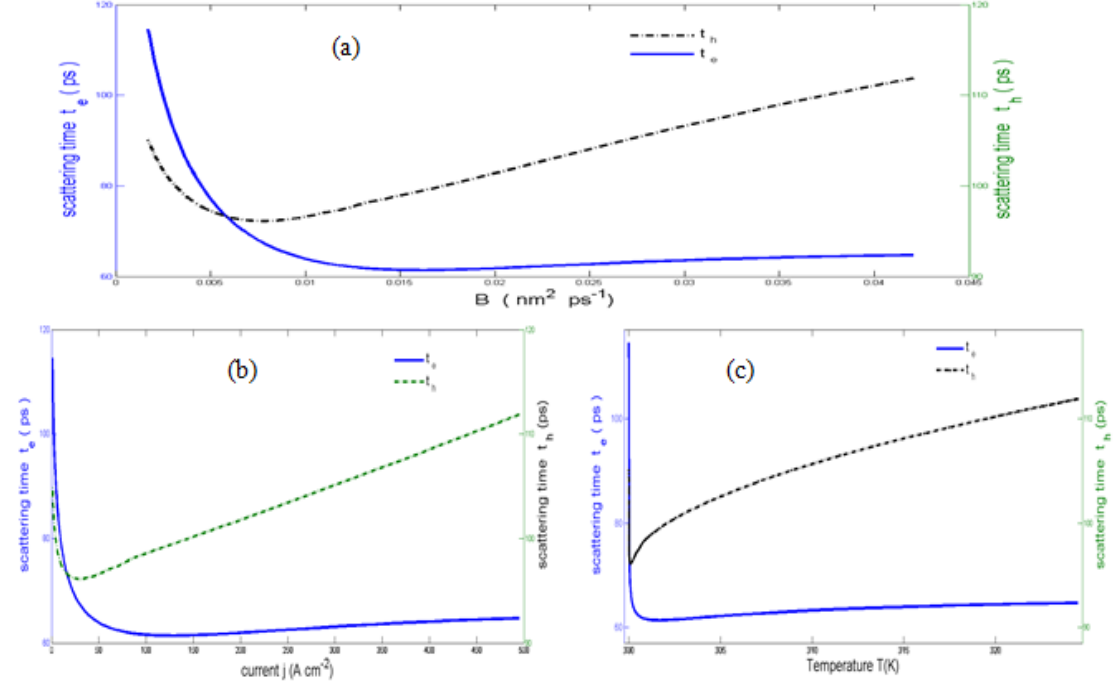

Fig (8) Simulation of the temporal variation of electrons and holes life times (microscopically calculated electron and hole scattering times $\tau_{e}$ and $\tau_{h}$ of the confined QD level) against (a) carrier reservoir loss coefficient $B,(\mathrm{~b})$ injection current density $(j)$ and (c)temperature $T(K)$.

\section{Dynamic Parameters:}

There are dynamical effects that occur with increasing injection current density. The shift of the device temperature inside an electrically pumped optical amplifier (with identical active region) affected by changing the injection current density. The functional relationship between the temperature shift and injection current density is given as $\Delta T(j) \sim j^{2}$. 
Since this temperature change is due to an increasing QW carrier density, and the QW carrier density itself depends via $w_{e} \sim \sqrt{j}$ [2] on the pump current, it implemented $\Delta T\left(w_{e}\right) \sim\left(w_{e}\right)^{4}$ as given by (9)

$$
T=300+0.245 \cdot 10^{12} \mathrm{~nm}^{8}\left(w_{\varepsilon}\right)^{4}
$$

The loss inside the QW can be written as $R_{\text {loss }}=B w_{e} w_{h}$ which can be implemented in the rate equations (4) and (5) [4]. The constant $B$ can be written as:

$$
\begin{aligned}
B & =B^{S}+B_{A} w_{\varepsilon} \\
& =0.03 \mathrm{~nm}^{2} \mathrm{ps}^{-1}+305 \mathrm{~nm}^{4} \mathrm{ps}^{-1}\left(\frac{T}{300}\right)^{4} w_{\varepsilon}
\end{aligned}
$$

Thus Auger coefficient $B_{A}$ has been shown to depend significantly on the temperature $T$ [8]. The dynamic parameters $\left(T\left(w_{e}\right), B\left(w_{e}\right)\right)$ depended on the pump current under $\mathrm{CW}$ operation. This effect of implementing according to equations (9) and (10).

As expected the temperature hence $B$ should increase with increase of injection current density as shown in Fig.(9).

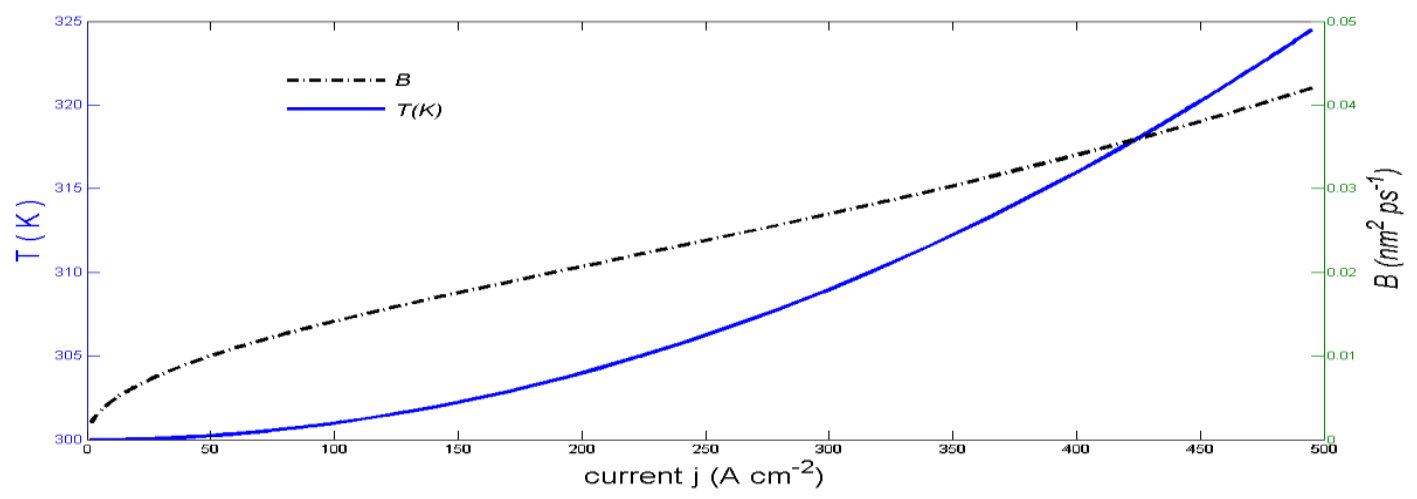

As the current density increased so does the temperature hence $B_{A}$ increased, las shown in Fig.(10.a) . $B_{A}$ depends on temperature which leads to

the increase in electrons and holes in the wetting layer, see(10.b). As the temperature increased the WL electron density increased too (Fig.(10.c)).
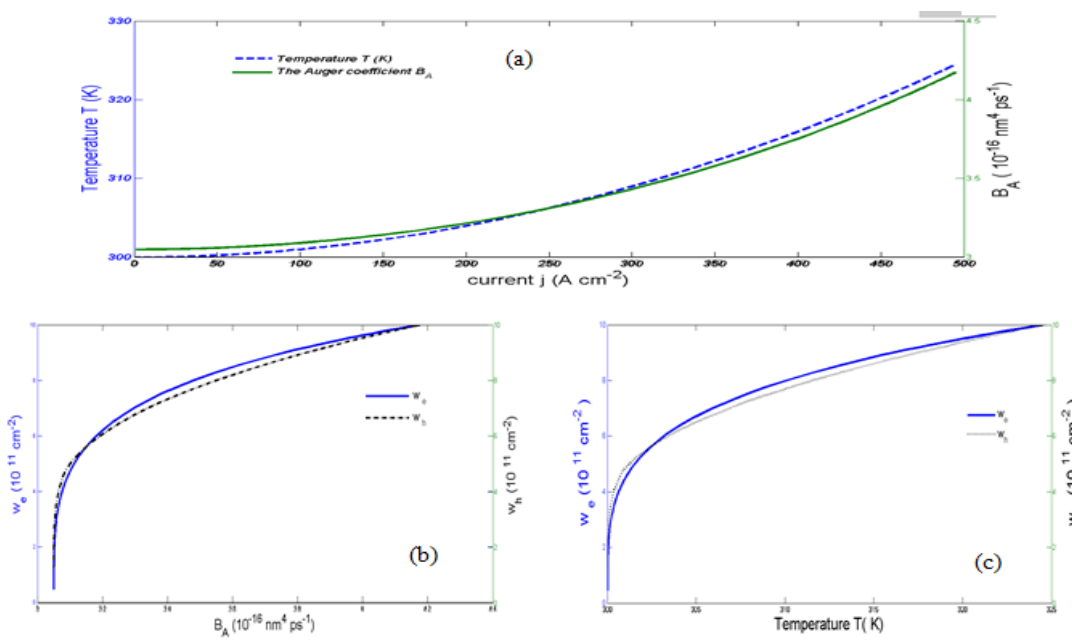

Fig (10).(a) The temporal variation of temperature $T$ and Auger coefficient $B_{A}$ at CW operation as a function of the pump current density $j,(\mathrm{~b}, \mathrm{c})$ Simulation of the temporal variation of WL- electron and hole density $\left(\left(w_{e}, w_{h)}\right)\right.$ against Auger coefficient $B_{A}$ and Temperature $T$, respectively . 


\section{Discussion}

Results given in Fig (5) can be explained as follows: for a certain injection current the number of electrons and holes in QD show fast increase followed by a peak then it settle down after the transient regime. The number of photons, follow the same behavior of the populations e and $\mathrm{h}$. As the temperature increases $w_{e}$ increased too (see equation (9)) .Since $w_{e}$ and $w_{h}$ show direct proportionality against injection current, the result of Fig $(6(a, b))$ of the $\left(S_{e}^{\text {in/out }}, S_{h}^{\text {in/out }}\right)$ can be explained as follows: the calculated scattering rates depend in a strongly nonlinear way upon the WL carrier densities [7]. The curves in Fig. 6(a) and (b) show the in- and outscattering rates for electrons and holes, respectively. Note that the values for electron and hole in-scattering rates differ by about a factor of 2 and the out-scattering rates differ by two orders of magnitude. For very low WL carrier densities, the in-scattering rate shows a quadratic increase as predicted by mass action kinetics, but it deviates from this functional relation for increasing WL carrier densities. The out-scattering is characterized by a sharp increase with increasing density of scattering partners followed by a decrease that is caused by Pauli blocking of the WL states. Since the holes have a larger effective mass, the maximum of the out-scattering rate lies at higher WL carrier densities than for electrons [2]. At the same time as $w_{e}$ increases scattering times increased too. These behaviors are shown in Fig. 7(c, d). $B_{A}$ depends strongly on temperature [1] so that scattering times shows behavior given in Fig.7 $(a, b)$ which is the same as those shown in Fig.(6). As temperature increases so does $\left(w_{e}\right)$; at the same time $B_{A}$ increased too. The relation between scattering times of electrons and holes ( $\tau_{e}$ and $\tau_{h}$ ) the carrier reservoir loss coefficient $(B)$ is equivalent to the reciprocal of the relation between scattering times and temperature and $w_{e}$ [2].The effects of $B$, current injection density(j) and temperature are shown in Fig(8). Fig (9) summarizes the effect of injection current on different parameters affecting the dynamics of QD laser via the effect of $j(t)$ on temperature.

\section{Conclusion}

Based on the work of Kathy Ludge, we have studied the dependence of different variables such photon density, in- and out- scattering rates, scattering times of electrons and holes, temperature and carrier loss coefficient, on number of parameters such injection current density,Auger coefficient. Results obtained agree with experimental result of other researchers $[2,7]$.

\section{References}

1. "Nonlinear laser dynamics ", Ed .K . Ludge ,Wiley $-\mathrm{VCH}$ Verlag $\mathrm{GmBH}$ and Co. KGaA ,Germany (2012) .

2. K. Lüdge and E. Schöll, "Quantum-dot lasersdesynchronized onlinear dynamics of electrons and holes," IEEE J.QE, 45, 1396- 1403, Nov. (2009).

3. N. Majer, K. Ludge and E. Scholl, Cascading enables ultrafast gain recovery dynamics of quantum dot semiconductor optical amplifiers ,Phys. Rev. B, 82, 235301-1-6(2010).

4. K .Ludge, R. Aust ,G. Fiol ,M. Stubenrauch ,D. Arsenijevi'c, D .Bimberg and E.Scholl, Large signal response of semiconductor quantumdot lasers, IEEE J.QE,46， 1755- 1761,(2010).

5. K. Lüdge and E. Schöll, "Nonlinear dynamics of doped semiconductor quantum dot lasers," Eur. Phys. J. D, 58,167-174, (2010).

6. E. Malic', M. J. P. Bormann, P. Hövel, M. Kuntz, D. Bimberg, A. Knorr, and E. Schöll, "Coulomb damped relaxation oscillations in semiconductor quantum dot lasers," IEEE J. Sel. Topics Quantum Electron., 13, 1242- 1248, (2007).

7. K. Lüdge, M. J. P. Bormann, E. Malic', P. Hövel, M.Kuntz, D. Bimberg,A. Knorr, and E. Schöll, "Turn-on dynamics and modulation response in semiconductor quantum dot lasers," Phys. Rev. B, 78, 035316-1-03531611,( 2008).

8. J. M. Pikal, C. S. Menoni, P. Thiagarajan, G. Y. Robinson, and H.Temkin, "Temperature dependence of intrinsic recombination coefficients in $1.3 \mu \mathrm{m}$ InAsP/InP quantum-well semiconductor lasers," Appl. Phys. Lett., 76, 2659-2661, ( 2000).

9. M. Rossetti, A.Fiore, G.Sek, , C.Zinoni, and L. Li, Modeling the temperature characteristics of InAs/GaAs quantum dot lasers, J. Appl. Phys., $106,023105,(2009)$. 